\title{
Assessment of intra-articular volume of the wrist: a comparative study between CT-arthrography and dissection
}

Received: 4 December 2004 / Accepted: 31 May 2005/Published online: 25 August 2005

(C) Springer-Verlag 2005

\begin{abstract}
The aim of this study was to compare the intraarticular volumes of the wrist's joint measured by CTarthrography and by dissection on ten cadavers. A good correlation was found between CT-arthrography and dissection in the assessment of the intra-articular volume of both wrist joints. A volume of $1.34( \pm 0.46)$ milliliter and $0.97( \pm 0.32)$ milliliter were found by CT-arthrography for the radiocarpal and midcarpal joints, respectively. Dissection of the same wrists gives a volume of $1.24( \pm 0.33)$ and $0.90( \pm 0.21)$ for the radiocarpal and midcarpal joints, respectively. The knowledge of normal wrist-joint volume is a major prerequisite to evaluate carpal instability without ligaments' tears. We believe that CT-arthrography could be helpful in evaluating patients with suspected carpal instability.
\end{abstract}

Keywords Wrist · Arthrography · CT-arthrography · Anatomy $\cdot$ Ligaments

X. Montet $(\bowtie)$

Division de radiodiagnostic et de radiologie interventionnelle, unité dímagerie numérique, Hôpital Universitaire de Genève, 24, rue Micheli-du-Crest,

1211 Geneva 14, Switzerland

E-mail: xavier.montet@hcuge.ch

Tel.: +41-22-3723311

Fax: + 41-22-3727072

J.-L. Eberlin

FMH chirurgie plastique reconstructive et esthétique,

27 route de Fontenais,

2900 Porrentruym, Switzerland

S. Bianchi

Fondation des Grangettes, $7 \mathrm{ch}$. des Grangettes,

1224 Chêne-Bougeries, Switzerland

J. Fasel

Département de Morphologie,

Centre médical universitaire de Genève,

Geneva, Switzerland

\section{Introduction}

Intrinsic wrist ligament tears are difficult to assess by clinical means and require imaging modalities for an accurate evaluation. Arthrography is a useful tool to detect complete tears that are diagnosed in the presence of communication among different joint compartments, which are separated in normal conditions. When injection of the dye is made inside a compartment, contrast diffusion in an adjacent joint is a definite proof of a fullthickness rupture. Arthrography is a minimally invasive procedure, with very low rates of complications [7]. New radiological techniques, such as spiral CT-arthrography or MR-arthrography are now available, allowing a more in depth assessment of wrist ligaments as well as of articular cartilages and adjacent soft tissues in the same examination. In some patients, wrist instability seems to depend more on ligament laxity since no ligament tears can be found in these patients. We believe that an increase in wrist-joint capacity can reflect ligament laxity and its measurement can be useful in the clinical assessment of patients without tears. In an effort to evaluate if arthro-CT evaluation of volumes of the different wrist compartments is reliable, we performed a prospective evaluation of ten wrists comparing CT findings with dissection data. The aim of this study was to measure the intra-articular volumes of the radiocarpal and midcarpals joint with CT-arthrography and compare them with data obtained from anatomic dissection.

One of the major prerequisites for interpretation of an arthrographic-type exam is a good knowledge of the wrist's ligaments. Hence, we will first discuss the anatomy of the ligament of the wrist and then compare the intra-articular volume of the radiocarpal and midcarpal joints as assessed by CT-arthrography and dissection. Finally, we will discuss the relationship between abnormal intra-articular wrist volume without clear ligament tears and carpal instability.

Anatomy of the ligament of the wrist (Fig. 1): The carpal ligaments are divided into extrinsic and intrinsic 
Fig. 1 Schematic illustration of the dorsal and volar ligament complex of the wrist. 1 radiotriquetral ligament, 2 dorsal intercarpal ligament, 3 radial collateral ligament, 4 radioscaphocapitate ligament, 5 radiolunotriquetral ligament, 6 radioscaphoid ligament, 7 radioscapholunate ligament, 8 radiolunate ligament, 9 ulnolunate ligament, 10 ulnotriquetral ligament
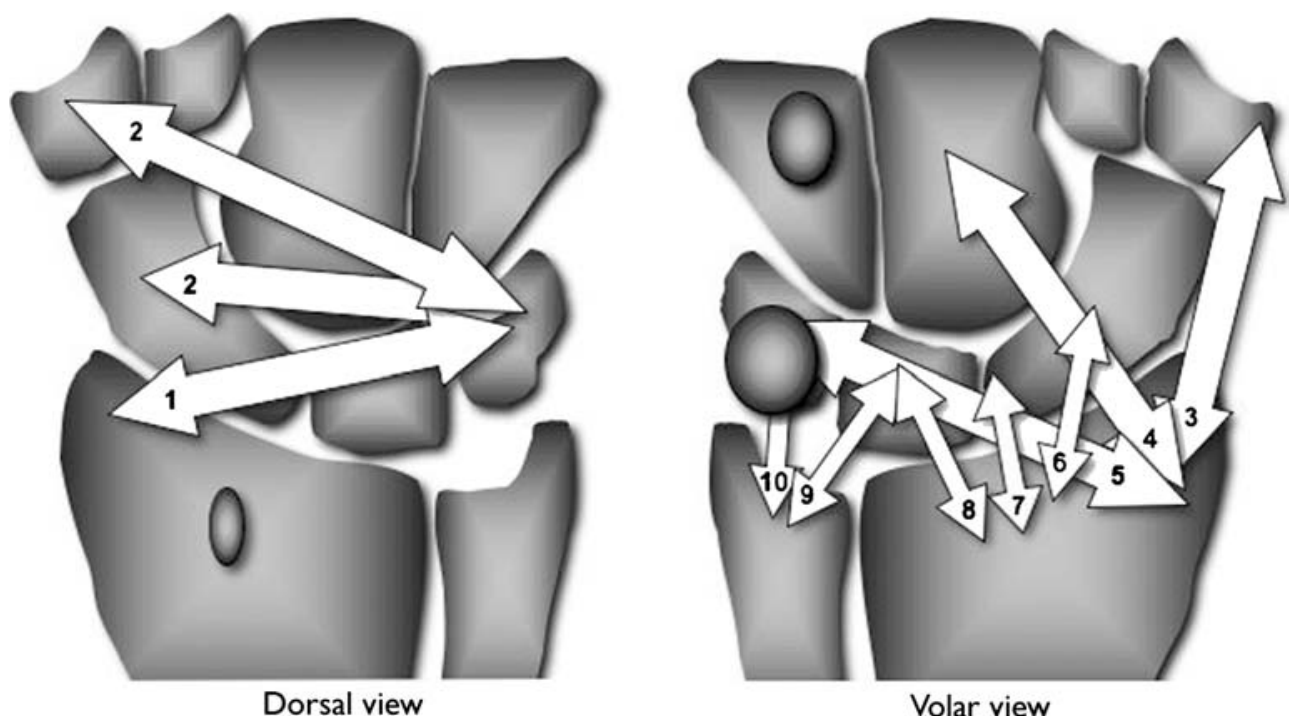

ligaments. By definition, extrinsic ligaments originate in the forearm and insert into the carpal bones while intrinsic ligaments originate from and insert into the carpal bones.

The dorsal ligament complex is formed by one extrinsic and one intrinsic ligament. The radiotriquetral ligament (RTL) belongs to the extrinsic ligaments; it arises from the dorsal radius near the Lister's tubercle and inserts into the dorsal part of the triquetrum and is formed by one to three fascicles. The dorsal intercarpal ligament (DIL) belongs to the intrinsic ligament. It is formed by two fascicles. Both fascicles arise from the dorsal triquetrum, but the triquetroscaphoid fascicle inserts into the dorsal part of the scaphoid and the triquetrotrapezial fascicle inserts into the palmar part of the trapezial.

The volar ligament complex is formed by eight ligaments, which can be considered as three functional groups. Three ligaments arising from the styloid process of the distal radius compose the first functional group, one to three ligaments arising from the radius form the second functional group and two ligaments arising from the triangular fibrocartilage form the third functional group.

The first functional group is formed by the radial collateral ligament (RCL), the radioscaphocapitate ligament (RSCL) and by radiolunotriquetral ligament (RLTL).

The second functional group is formed by the radioscaphoid ligament (RSL), the radioscapholunate ligament (RSLL) and the radiolunate ligament (RLL).

The third functional group is formed by the ulnolunate ligament (ULL) and by the ulnotriquetral ligament (UTL). Intercarpal (intrinsic) ligaments (Fig. 2).

These ligaments formed the boundaries of the radiocarpal and midcarpal joints. The scapholunate (SLL) and the lunotriquetral ligaments (LTL) formed together with the bones the limits of the radiocarpal joint. The scaphotrapeziotrapezoid (STTL), trapezoicapitate
(TCL) and capito-hamate ligaments (CHL) formed together with bones the limits of the midcarpal joint.

The two most clinically important intrinsic ligaments of the wrist are the SLL and lunotriquetral [10]. Both ligaments have volar and dorsal segments strongly attached to the carpal bones and the extrinsic ligaments.

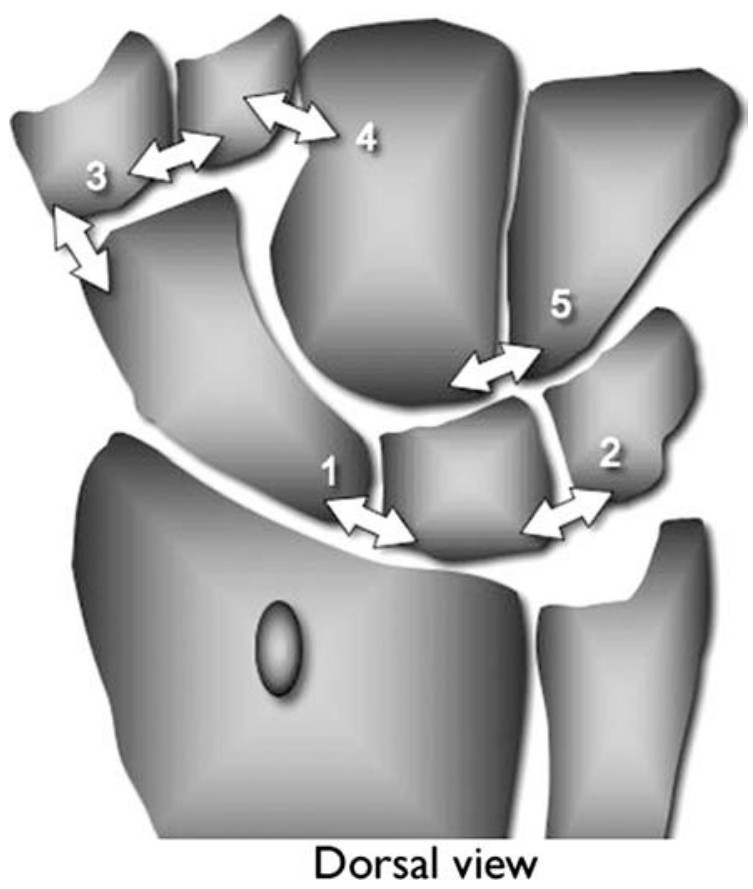

Fig. 2 Schematic illustration of the intercarpal (intrinsic) ligaments of the wrist. The scapholunate (1) and the lunotriquetral ligaments (2) formed together with the bones the limits of the radiocarpal joint. The scaphotrapeziotrapezoid (3), trapezoicapitate (4) and capito-hamate ligaments (5) formed together with bones the limits of the midcarpal joint 


\section{Materials and methods}

Ten fresh-frozen human wrists were utilized in this study. Due to anonymization of the anatomical pieces, we had no clinical data concerning these wrists. Institutional guidelines for work with anatomical specimens were followed during this protocol. The specimens were derived from arms cut through the middle portions of the radius and ulna. They were thawed at room temperature for 12-24 h before arthrography.

Arthrography was performed with a compound made with $10 \%$ iodinated contrast media (Isovist 300), 90\% plastic polymer, as well as a drop of methylene blue.
Under fluoroscopic guidance, a 20-gauge needle was directly inserted by a dorsal approach into the midcarpal and subsequently the radiocarpal joint. The needle position was verified with a test injection of a small amount of iodinated polymer. The volume of iodinated polymer injected in each joint was recorded. To precisely record the injected volume into each articulation, we used a 3$\mathrm{ml}$ syringe, with $1 / 10-\mathrm{ml}$ graduation and the volume injected in each articulation was noted. Conventional postero-anterior radiographs were then acquired. The CT arthrography was performed between 1 and $24 \mathrm{~h}$ after dye injection. Axial images were obtained with a multi-slice spiral CT Philips MX 8000 (Philips Medical System, The Netherlands) with the following parame-
Fig. 3 Images obtained during arthrography. Early and complete filling of the radiocarpal joints are presented in $\mathbf{a}$ and $\mathbf{b}$, respectively. Early and complete filling of the midcarpal joints are presented in $\mathbf{c}$ and $\mathbf{d}$, respectively. Each joint was filled with a mixture of polymer and iodine. The iodine was used to allow visualization of the joint during arthrography and CT-arthrography. The polymer was used to assess the volume of the joints during anatomical dissection
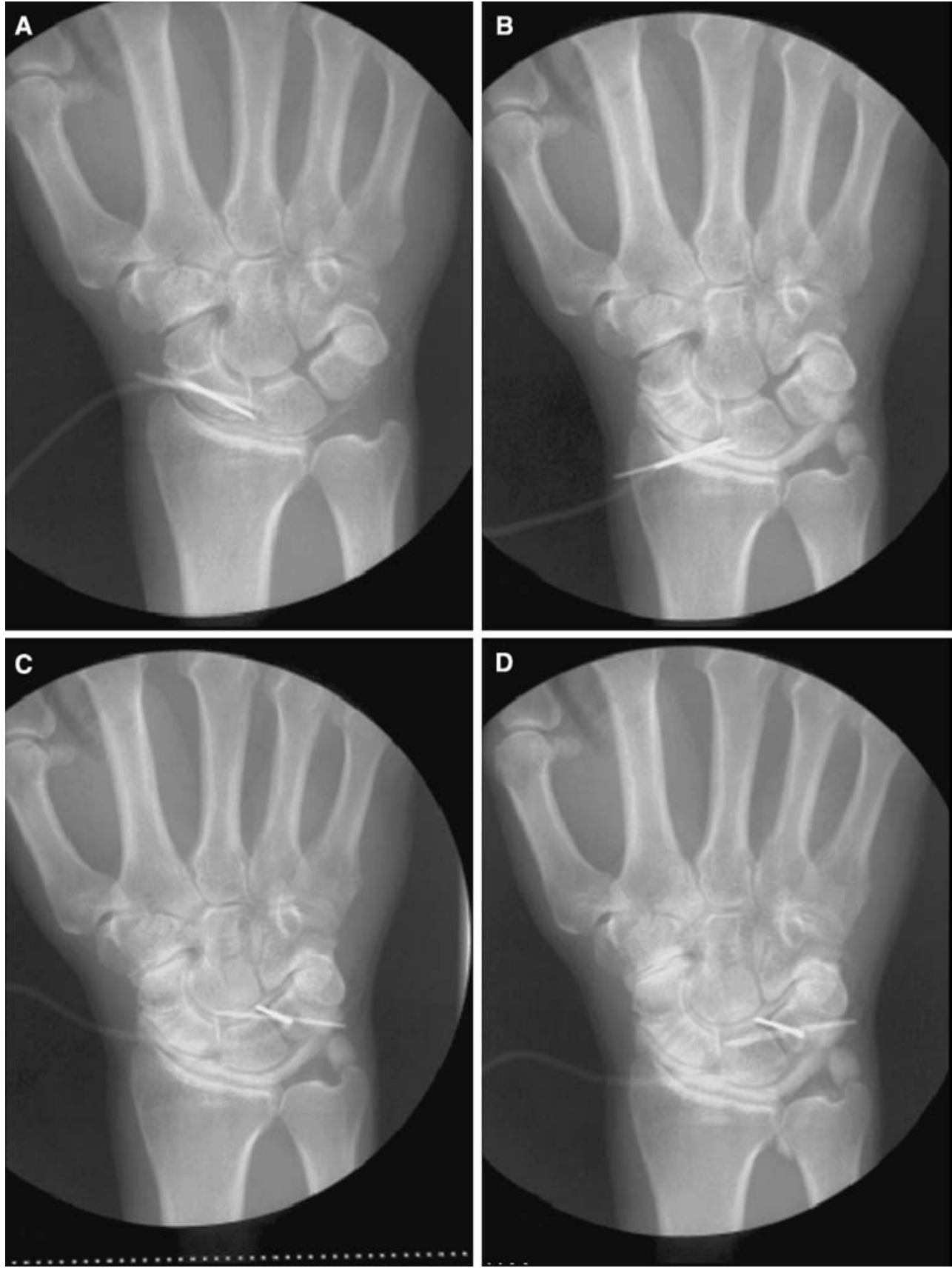
ters: slice thickness $=1.3 \mathrm{~mm}$, pitch $=0.6 \mathrm{~mm}, \mathrm{KV}=120$, $\mathrm{mA}=125$, FOV $12-15 \mathrm{~cm}$ (according to the size of the wrist), matrix size $=512 \times 512$. All wrists were imaged in prone position. Post-acquisition volume rendering, as well as intra-articular volume, were measured on a Vitrea workstation (Vital Image, Minneapolis, MN, USA).

For volume measurements regions of interest (ROI) were defined in the radiocarpal and the midcarpal joints. These ROI were defined in the reconstructed coronal plane obtained from the native axial images. Multiple ROI were traced, at $1 \mathrm{~mm}$ of interval, from the most anterior part of the joint to the posterior border. The computer then adds the ROI between them and calculates the volume of each joint in milliliter.

After CT specimens were placed in $4 \%$ formol to allow a good polymerization of the injected compound. The formol allowed good specimen preservation permitting good quality dissections that were performed within 3 days. An experienced surgeon dissected all wrists and the volumes of the intra-articular polymer were assessed using the Archimed principle. Dissection was performed with the wrist placed on a table, with the palmar side down. The dorsal soft tissues, which were adjacent to the radiocarpal and midcarpal joints, were removed. Then, the dorsal ligaments were identified and the presence of extra-articular blue iodinated polymer, if any, noted. In the case of extra-articular iodinated polymer, this was removed before entering the joint, thus allowing measurements of only the intra-articular volume. Then, the radiocarpal joint was opened and the iodinated polymer removed and placed in a graduated cylinder containing water. The displaced water volume that corresponded to the volume of polymer was measured with a precision of $1 / 10 \mathrm{ml}$. The same procedure was applied for the midcarpal joint.

The CT-arthrography results and the dissection data were statistically compared. All the values are given in mean $( \pm \mathrm{SD})$. A Student $t$-test was applied with a $p$ value $<0.05$ considered significant.

\section{Results}

All contrast injections were of good quality, as assessed by images obtained during arthrography and the CTarthrography (Figs. 3, 4). The volume of injected iodinated polymer as well as the volume found by CTarthrography and dissection are summarized in Table 1 .
Fig. 4 CT-arthrography of the wrist. The reformatted coronal view was used to draw the limit of the radiocarpal and midcarpal joint. Delimitation of the midcarpal joint (a), as well as the $3-\mathrm{D}$ reconstruction (b) are presented
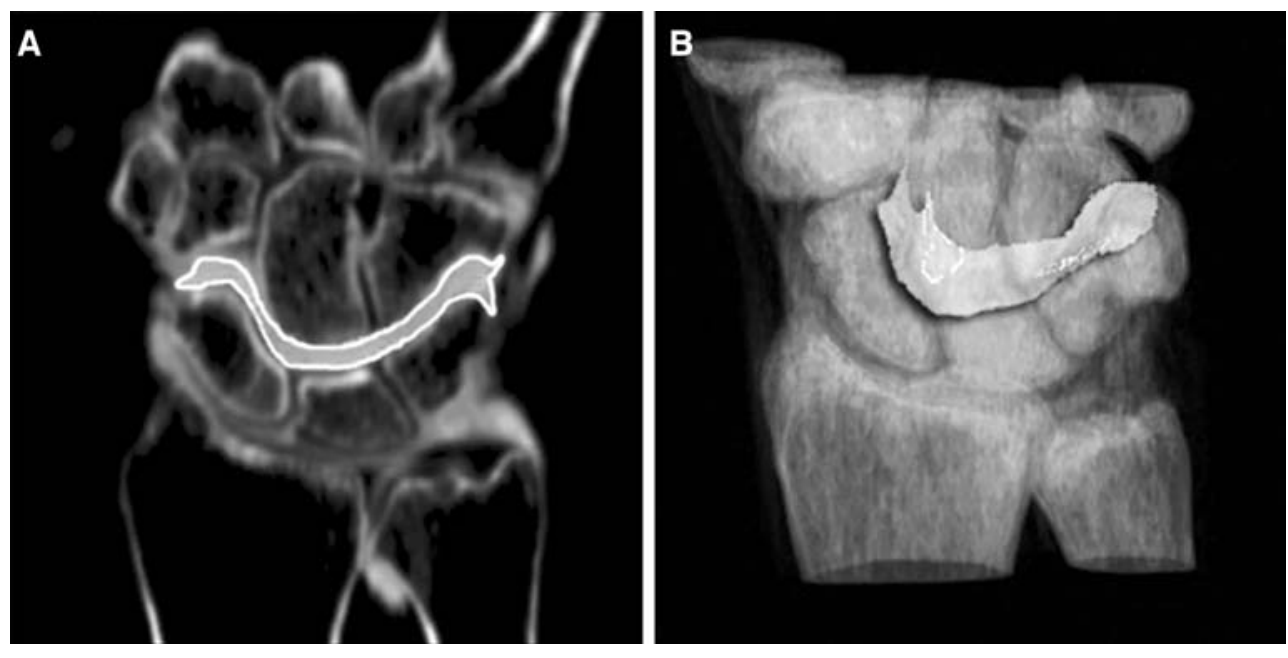

Table 1 Comparison of the volume of radiocarpal and midcarpal joints found by arthrography, CT-arthrography and dissection

\begin{tabular}{|c|c|c|c|c|c|c|}
\hline & \multicolumn{2}{|c|}{ Arthrography (Volume in milliliter) } & \multicolumn{2}{|c|}{$\begin{array}{l}\text { CT-Arthrography (Volume in millili- } \\
\text { ter) }\end{array}$} & \multicolumn{2}{|c|}{ Anatomy (Volume in milliliter) } \\
\hline & Radiocarpal joint & Midcarpal joint & Radiocarpal joint & Midcarpal joint & Radiocarpal joint & Midcarpal joint \\
\hline Specimen 1 & 1.8 & 1.0 & 1.5 & 1.0 & 1.5 & 1.0 \\
\hline Specimen 2 & 2.5 & 2.0 & 1.2 & 1.2 & 1.5 & 1.2 \\
\hline Specimen 3 & 2.2 & 1.7 & 1.1 & 1.1 & 1.0 & 1.2 \\
\hline Specimen 4 & 1.8 & 1.5 & 1.3 & 0.8 & 1.2 & 0.8 \\
\hline Specimen 7 & 1.5 & 1.5 & 1.3 & 1.0 & 1.5 & 1.1 \\
\hline Specimen 8 & 2.5 & 1.5 & 2.3 & 1.1 & 2.0 & 0.9 \\
\hline Specimen 9 & 1.5 & 1.0 & 1.2 & 0.9 & 1.5 & 1.0 \\
\hline Specimen 10 & 1.5 & 1.0 & 1.1 & 1.0 & 1.5 & 0.7 \\
\hline
\end{tabular}




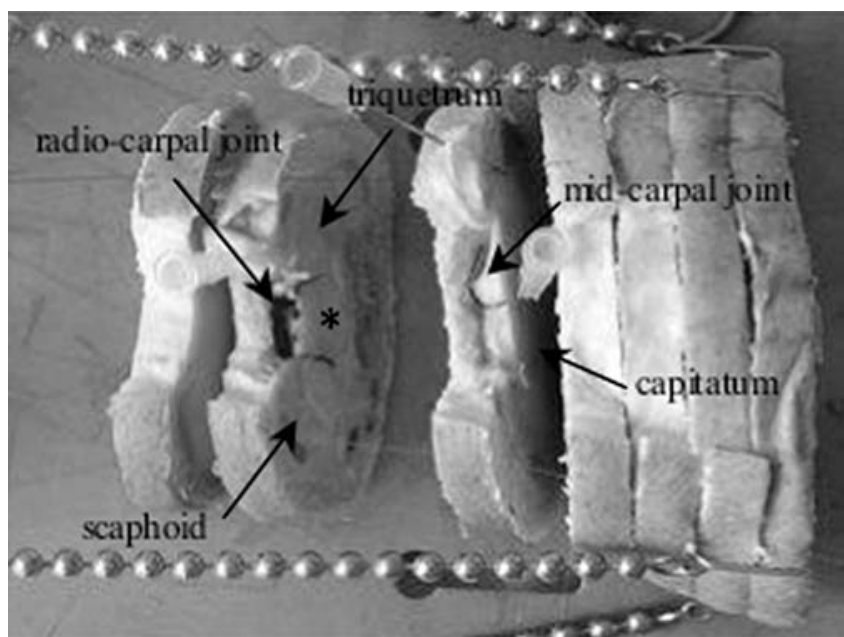

Fig. 5 Anatomical dissection of the wrist. Dissection of the wrist allows emptying the radiocarpal and midcarpal joint. Once the polymer was retrieved from each joint, its volume was measured. This volume was compared with the volume found by CTarthrography. *Lunatum

The dissection allows good access to both radiocarpal and midcarpal joints (Fig. 5). On two anatomical pieces, a direct communication between the radiocarpal and midcarpal joints were noted on the ulno-dorsal side (wrists 7 and 8); in three cases, tears of radiocarpal ligaments with extravasations of polymer were noted (wrists 2, 4 and 9); in one case, extravasations of polymer were noted distal to the midcarpal ligaments, without clear ligament tears (wrist number 3 ).

The 3-D reconstruction of the CT-arthrography of the radiocarpal joint gives a value of $1.34( \pm 0.46)$ milliliter. The same reconstruction for the midcarpal joint gives values of $0.97( \pm 0.32)$ milliliter.

The dissection of the joints gives a volume of 1.24 $( \pm 0.33)$ and of $0.90( \pm 0.21)$ for the radiocarpal and midcarpal joint, respectively. These volumes were not statistically different $(p>0.21$ and 0.25 for radiocarpal

Fig. 6 Linear regression between volume found during dissection and volume calculated in CT-arthrography. A good correlation was found between both techniques. The CT-arthrography is a useful tool to assess the intraarticular volume of the wrist. $90 \%$ confidence band

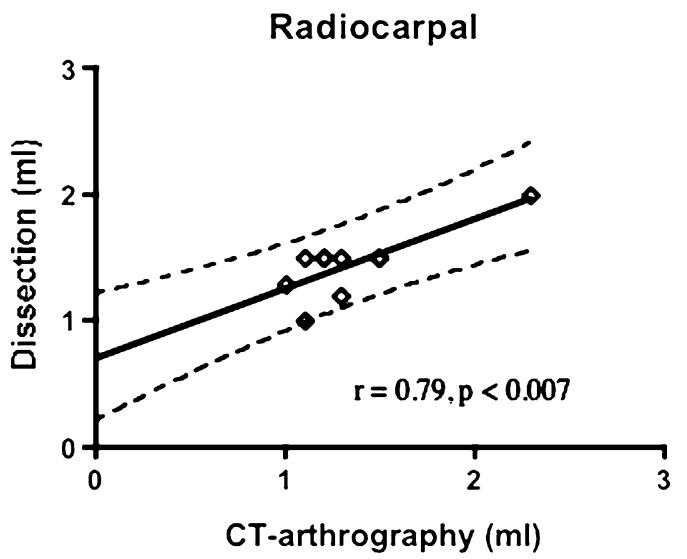

and midcarpal joint, respectively). A good correlation was found between CT-arthrography and dissection (Fig. 6).

\section{Discussion}

Although the normal anatomy of the dorsal wrist ligaments is well known to anatomists and hand surgeons [1], the implication of these ligaments in the biomechanics and stability of the hand and wrist is a new concept emerging in the 20 last years $[1,2,4,8,9]$. Numerous studies demonstrated the value of arthrographic modalities including standard arthrography [5], CT [3] or MRI-arthrography [3,6] in the detection of ligament tears, but to the best of our knowledge, no authors investigate precisely the normal intra-articular volume of the joints of the wrist.

These measures can have clinical applications. We postulate that if the intra-articular volume is superior to the normal value in patients without evidence of other causes of joint effusions, a ligament laxity can be present and can cause dynamical dysfunction of the wrist, because the stability of the carpal bones is no more adequate. The volume of wrist joint is classically evaluated by arthrography, an imaging modality that needs a joint puncture and intra-articular injection of contrast dye. Although arthrography is a safe modality, an intraarticular needle positioning through a dorsal wrist approach is necessary and can be seldom painful. In addition, utilization of iodinated contrast must be limited in allergic patients. In an effort to investigate if CT can be useful in determining the amount of effusion located within the different wrist joints, we have evaluated CT data and correlated them with anatomic data. In our study, the values were 1.5 and $1 \mathrm{ml}$ for the normal radiocarpal and midcarpal joint, respectively.

Presence of ligament tears did not represent a limitation in our study since the intra-articularly injected compound underwent polymerizing inside the joint cavity and accurate cadaveric dissection allowed removing the extra-articular compound.

Our study shows a good correlation between intraarticular volume evaluated by CT-arthrography and cadaveric study. The absolute values we obtained are

\section{Midcarpal}

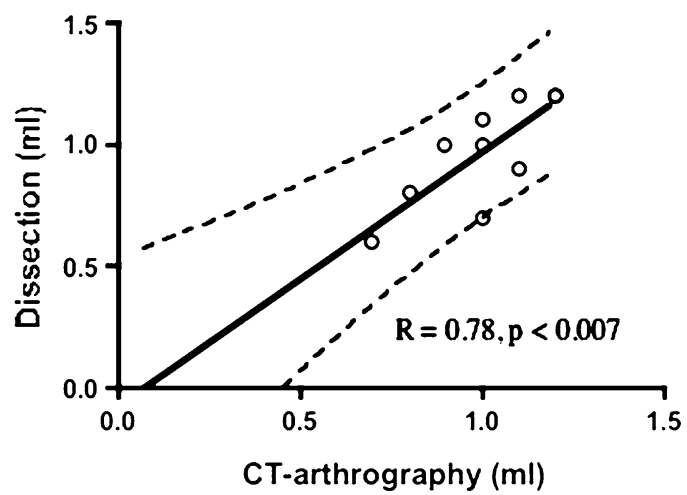


probably underestimated with respect to normal volumes in vivo, because of cadaver retraction and/or rigidity. Nevertheless, since values between CT and cadaver dissections correlated well we can postulate a similar correlation in vivo. Although we can only speculate on that, we believe that the same accuracy could be observed with CT of patients presenting with an effusion of the wrist joints. Hopefully, the measurement can suggest ligament hyperlaxity in patients in whom other causes of fluid accumulation have been excluded.

We strongly believe that the normal intra-articular volume of wrist joints have to be known to asses carpal instability without ligament tears. The same type of study has to be made in a clinical situation to have normal intra-articular volume of wrist joints in patients and to correlate it with ligament laxity.

\section{Conclusion}

This study demonstrated a good correlation between volumes of the radiocarpal and midcarpal joints calculated by CT-arthrography and by cadaveric dissections. We believe that, in the absence of other causes of joint effusions, increased intra-articular wrist joint volumes must be retained as pathologic even in the absence of evidence of ligament tears and that can reveal ligament laxity, responsible for carpal instability. Further studies are needed to evaluate the role of CT-arthrography in the diagnosis of ligament laxity and carpal instability without ligament tears.

\section{References}

1. Fahrer M (1980) Traité de chirurgie de la main tome 1:166

2. Kapanji IA (1998) La main 4:417-428

3. Klein HM, Vrsalovic V, Balas R, Neugebauer F (2002) Imaging diagnostics of the wrist: MRI and Arthrography/Arthro-CT. Rofo Fortschr Geb Rontgenstr Neuen Bildgeb Verfahr 174:177-182

4. Kuhlmann N (1977) Revista espagnola de la cirurgia de la mano 5:12-104

5. Linkous MD, Gilula LA (1998) Wrist arthrography today. Radiol Clin North Am 36:651-672

6. Meier R, Schmitt R, Christopoulos G, Krimmer H (2002) Scapholunate ligament tears in MR arthrography compared with wrist arthroscopy. Handchir Mikrochir Plast Chir 34:381385

7. Newberg AH, Munn CS, Robbins AH (1985) Complications of arthrography. Radiology 155:605-606

8. Sennwald GR, Zdravkovic V, Kern HP, Jacob HA (1993) Kinematics of the wrist and its ligaments. J Hand Surg [Am] 18:805-814

9. Sennwald GR (1999) The effects of wrist distraction on carpal kinematics. J Hand Surg [Am] 24:1344-1345

10. Viegas SF, Patterson RM, Hokanson JA, Davis J (1993) Wrist anatomy: incidence, distribution, and correlation of anatomic variations, tears, and arthrosis. J Hand Surg [Am] $18: 463-475$ 